\title{
Prognostic factors and treatment of neuroendocrine tumors of the uterine cervix based on the FIGO 2018 staging system: A single-institution study of 172 patients
}

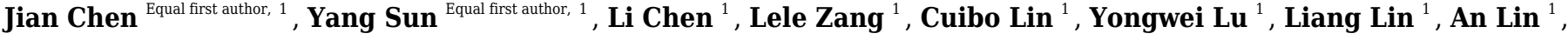 \\ Hu Dan ${ }^{2}$, Yiyu Chen ${ }^{2}$, Haixin He ${ }^{\text {Corresp. } 1}$ \\ ${ }^{1}$ Department of Gynecological-Surgical Oncology, Fujian Medical University Cancer Hospital, Fujian Cancer Hospital, Fuzhou, Fujian, China \\ 2 \\ 2 Department of Pathology, Fujian Medical University Cancer Hospital, Fujian Cancer Hospital, Fuzhou, Fujian, China \\ Corresponding Author: Haixin He \\ Email address: 63804657@qq.com
}

Objective: This study aimed to explore the prognostic factors and outcomes of patients with neuroendocrine tumors (NETs) of the cervix and to determine appropriate treatment.

Methods: A single-institution retrospective analysis of 172 patients with NETs was performed based on the new International Federation of Gynecology and Obstetrics (FIGO 2018) staging system.

Results: Among the 172 eligible patients, 161 were diagnosed with small cell neuroendocrine carcinoma (SCNEC), 6 with large cell neuroendocrine carcinoma, 4 with typical carcinoid tumor tumors and 1 with SCNEC combined with an atypical carcinoid tumor. According to the FIGO 2018 staging guidelines, 31 were stage I, 66 were stage II, 57 were stage III, and 18 were stage IV. The five-year survival rates of patients with stage I, II, III, and IV disease were $74.8 \%, 56.2 \%, 41.4 \%$ and $0 \%$, respectively. The five-year progression-free survival rates of patients with stage I, II, III, and IV disease were $63.8 \%, 54.5 \%, 30.8 \%$ and $0 \%$,respectively. In the multivariate analysis, advanced FIGO stage, large tumor and older age were identified as independent variables for five-year survival in patients with stage I-IV disease. FIGO stage, tumor size and para-aortic lymph node metastasis were independent prognostic factors for five-year progression-free survival in patients with stage I-IV disease. For the patients receiving surgery $(n=108)$, tumor size and pelvic lymph node metastasis were independent prognostic factors for five-year survival, and pelvic lymph node metastasis for five-year progression-free survival. In stage IVB, at least 6 cycles of chemotherapy $(n=7)$ was associated with significantly better 2 -year OS (83.3\% versus $9.1 \%, p \square 0.001)$ and 2 -year PFS $(57.1 \%$ versus $0 \%, p=0.01)$ than fewer than 6 cycles of chemotherapy $(n=11)$.

Conclusion: Advanced FIGO stage, large tumor, older age and lymph node metastasis are independent prognostic factors for NETs of the cervix. The TP/TC and EP regimens were the most commonly used regimens, with similar efficacies and toxicities. Standardized and complete multimodality treatment may improve the survival of patients with NETs. 


\section{Prognostic factors and treatment of neuroendocrine}

2 tumors of the uterine cervix based on the FIGO 2018

3 staging system:A single-institution study of 172

4 patients

5

6

7

8
${ }^{1}$ Department of Gynecological-Surgical Oncology, Fujian Medical University Cancer Hospital, Fujian Cancer Hospital, Fujian, China

2 Department of Pathology, Fujian Medical University Cancer Hospital, Fujian Cancer Hospital, Fujian 350014, China

a.The first two authors contributed equally to this article.

Corresponding Author:

HaiXin $\mathrm{He}^{1}$

Gynecological-Surgical Oncology, Fujian Medical University Cancer Hospital, Fujian Cancer Hospital, Fuzhou 350014, China

Email address: 63804657@qq.com

Jian Chen ${ }^{1, a}$,Yang Sun ${ }^{1, a}$, Li Chen ${ }^{1}$, Lele Zang ${ }^{1}$, CuiBo Lin ${ }^{1}$,YongWei Lu ${ }^{1}$, Liang Lin ${ }^{1}$, An $\mathrm{Lin}^{1}$, Dan $\mathrm{Hu}^{2}$, Yiyi Chen ${ }^{2}$, Haixin $\mathrm{He}^{1,{ }^{*}}$. 
36

37

38

39

40

41

42

43

44

45

46

47

48

49

50

51

52

53

54

55

56

57

58

59

60

61

62

63

64 65

66

\section{4}

\section{Abstract}

Objective: This study aimed to explore the prognostic factors and outcomes of patients with neuroendocrine tumors (NETs) of the cervix and to determine appropriate treatment.

Methods: A single-institution retrospective analysis of 172 patients with NETs was performed based on the new International Federation of Gynecology and Obstetrics (FIGO 2018) staging system.

Results:Among the 172 eligible patients, 161 were diagnosed with small cell neuroendocrine carcinoma (SCNEC), 6 with large cell neuroendocrine carcinoma, 4 with typical carcinoid tumor tumors and 1 with SCNEC combined with an atypical carcinoid tumor. According to the FIGO 2018 staging guidelines, 31 were stage I, 66 were stage II, 57 were stage III, and 18 were stage IV. The five-year survival rates of patients with stage I, II, III, and IV disease were $74.8 \%$, $56.2 \%, 41.4 \%$ and $0 \%$, respectively. The five-year progression-free survival rates of patients with stage I, II, III, and IV disease were $63.8 \%, 54.5 \%, 30.8 \%$ and $0 \%$,respectively. In the multivariate analysis, advanced FIGO stage, large tumor and older age were identified as independent variables for five-year survival in patients with stage I-IV disease. FIGO stage, tumor size and para-aortic lymph node metastasis were independent prognostic factors for fiveyear progression-free survival in patients with stage I-IV disease. For the patients receiving surgery $(n=108)$, tumor size and pelvic lymph node metastasis were independent prognostic factors for five-year survival, and pelvic lymph node metastasis for five-year progression-free survival. In stage IVB, at least 6 cycles of chemotherapy $(n=7)$ was associated with significantly better 2-year OS $(83.3 \%$ versus $9.1 \%, p<0.001)$ and 2-year PFS $(57.1 \%$ versus $0 \%, p=0.01)$ than fewer than 6 cycles of chemotherapy $(n=11)$.

Conclusion: Advanced FIGO stage, large tumor, older age and lymph node metastasis are independent prognostic factors for NETs of the cervix. The TP/TC and EP regimens were the most commonly used regimens, with similar efficacies and toxicities. Standardized and complete multimodality treatment may improve the survival of patients with NETs. 


\section{Introduction}

68 Neuroendocrine cervical tumors (NETs) are a rare but highly aggressive form of cervical 69 cancer(Gardner et al. 2011; Satoh et al. 2014). NETs are divided into four categories, typical 70 carcinoid tumors, atypical carcinoid tumors (ACTs), Small cell carcinomas (SCNECs), and large cell neuroendocrine carcinomas (LCNECs), according to World Health Organization classifications. Well-differentiated typical and atypical carcinoids are categorized as low-grade neuroendocrine tumors (LGNETs), and the small and large cell types are categorized as highgrade neuroendocrine carcinomas (HGNECs). Small cell carcinoma is the most common type of NET, yet it accounts for less than 5\% of all cervical carcinomas(Albores-Saavedra et al. 1997;

76

77

78

79

80 Viswanathan et al. 2004). Because of the rarity of NETs, most studies on NETs have been perfomed on small samples or are case reports(Hoskins et al. 2003; Zivanovic et al. 2009). Therefore, the prognostic factors and treatment of patients with NETs are still controversial. We performed a retrospective review of 172 patients to analyze the clinicopathologic behaviors and prognostic factors of patients with NETs and to determine appropriate treatment guidelines. Lymph node metastasis was included in the revised 2018 International Federation of Gynecology and Obstetrics (FIGO) cervical cancer staging system. We evaluated the prognosis of NETs according to the FIGO 2018 staging system. We also evaluated the benefit of neoadjuvant therapy, adjuvant chemotherapy and adjuvant radiotherapy. Finally, we discuss the appropriate chemotherapy regimen and number of cycles for NETs.

\section{Materials \& Methods}

\subsection{Patients and data collection}

This was a retrospective study. After obtaining approval from the Ethics Committee of Fujian Cancer Hospital(Ethical Application Ref: YKT2020-012-01), we reviewed the clinicopathological data of 195 patients with histologically confirmed NETs, who were diagnosed and treated at the Department of Gynecology of Fujian Cancer Hospital between November 2002 and June 2019. All patients signed informed consent. Those who had incorrect pathology report or lacked follow-up data were excluded from the study. Histologic slides were reviewed by two pathologists specialized in gynecological cancers to confirm the diagnosis of neuroendocrine tumors of the uterine cervix by a central pathological review(CPR).

\subsection{Central pathological review}


98

99

100

101

102

103

104

105

106

107

108

109

110

111

112

113

114

115

116

117

118

119

120

121

122

123

124

125

126

127

128

The CPR was performed by two pathologists specializing in gynecological cancers. Biopsy specimens were obtained from patients who started treatment with neoadjuvant therapy and who did not undergo surgery, whereas hysterectomy specimens were obtained from patients who underwent surgery. The pathologists had a consensus on the criteria for the diagnosis of NETs according to World Health Organization classifications. Carcinoid tumors show prominent nucleoli with the nested, island, organoid, spindled, or trabecular pattern and are characterized by abundant cytoplasm, and characteristic granular chromatin. The differences between atypical carcinoid and carcinoid tumors are a greater degree of nuclear atypia and mitotic activity $(5-10 / 10$ HPF) as well as rare areas of necrosis. SCNEC is composed of ovoid, poorly cohesive cells, with condensed chromatin and scant cytoplasm. There is frequent nuclear molding, numerous mitotic figures, necrosis and apoptotic bodies. The growth pattern may be diffuse, trabecular, nested or exhibit rosette-like structures(Fig.1.A). LCNECs are recognized by their arrangement in well demarcated nests, trabeculae or cords with peripheral palisading. Tumor cells are large and polygonal, with vesicular or hyperchromatic nuclei and a prominent nucleolus. There is high mitotic activity and extensive geographic necrosis(Fig.1.B). Immunohistochemical staining for neuroendocrine markers, including CD56, chromogranin A or synaptophysin was performed to confirm neuroendocrine features(Fig.1.C\&D). Nevertheless, positive neuroendocrine markers were not necessary for diagnosis(Conner et al. 2002). Mixed tumors were defined by the presence of squamous or glandular.

\subsection{Treatment variables}

The variables included age at diagnosis, tumor size, FIGO stage, pathological type, pure or mixed histology, lymph node metastasis, lymphovascular invasion (LVSI), parametrial involvement, perineural invasion, depth of stromal invasion, treatment and chemotherapy regimens and courses. lymph node metastasis was defined by histology in patients who underwent surgery, and by imaging studies, such as magnetic resonance imaging (MRI), computed tomography $(\mathrm{CT})$, or positron emission computed tomography (PET), in patients who started treatment with neoadjuvant therapy or did not receive surgery.

We divided treatment into seven categories, surgery alone, surgery with adjuvant treatment, surgery preceded by neoadjuvant chemotherapy with or without adjuvant treatment, chemotherapy alone, radiotherapy alone, radiotherapy with chemotherapy and concurrent chemoradiotherapy. 
129

130

131

132

133

134

135

136

137

138

139

140

141

142

143

144

145

146

147

148

149

150

151

152

153

154

155

156

157

158

159

\subsection{Statistical Analysis}

The probabilities of overall survival (OS) and progression-free survival (PFS) were estimated using the Kaplan-Meier method. The Cox proportional hazards model was used to identify prognostic factors. Prognostic factors with $\mathrm{P}$ values $<0.1$ in univariable analysdis were further assessed in multivariable analysis. The hazard ratios (HR) and 95\% confidence intervals (CI) were calculated using the Wald test.P values $<0.05$ were considered statistically significant. The SPSS 24.0 statistical package (SPSS Inc., Chicago, IL, United States) was used for statistical analysis.

\section{Results}

\subsection{Clinicopathologic characteristics of the patients}

A total of 195 patients diagnosed with cervical neuroendocrine carcinoma were enrolled in our study between November 2002 and June 2019. The reasons for exclusion were as follows: 3 patients were diagnosed with other histology after the CPR, 8 patients refused or discontinued treatment, 5 patients had insufficient medical information, and 7 were lost to follow-up. Ultimately, 172 patients were enrolled in our study (Fig.2).

The ages of the 172 enrolled patients ranged from 25 to 86 years, and the mean age was 46.7 years. The median cervical tumor dimension was $4.5 \mathrm{~cm}$. There were 161 cases of small cell carcinoma, 6 cases of large cell carcinoma and 4 cases of typical carcinoid tumor tumors. One patient was classified as having a "NET, not classified" because both SCNEC and ACT components were present. Pure histology was documented in $73.3 \%(126 / 172)$ of the patients (Table 1). The mixed histology pattern was associated mostly with adenocarcinoma (29/172 patients; 16.7\%). The remaining specimens were squamous cell carcinoma (14/172 patients; $8.1 \%$ ) and adenosquamous carcinoma (3/172 patients; $1.7 \%$ ). The median follow-up time was 50.7 months (range, 2-193).

Of the 172 patients, 36 had stage I disease, 101 had stage II disease, 17 had stage III disease, and 18 had stage IV disease, based on the FIGO 2009 staging system. When the FIGO 2018 staging system was used, 31 patients were classified as stage I, 67 as stage II, 56 as stage III, and 18 as stage IV. Based on lymph node metastasis, 5 patients were classified as stage I, 34 patients as stage II, and 1 patient as stage IIIA were upstaged to stage IIIC according to the FIGO 2018 staging system. In this series, 108 patients received surgery as their primary treatment, and 53 
160 patients received radiotherapy as their primary treatment. Among the 108 patients who received 161 surgery, 105 received radical hysterectomy with pelvic lymph adenectomy and 3 received only

162

163

164

165

166

167

168

169

170

171

172

173

174

175

176

177

178

179

180

181

182

183

184

185

186

187

188

189

190

simple hysterectomy without lymph node dissection. Overall $(\mathrm{n}=172), 76$ received etoposide and cisplatin/carboplatin (EP), 79 received paclitaxel and cisplatin/carboplatin (TP/TC) and 12 received the following regimens: 1 patient received paclitaxel liposomes, 2 received gemcitabine and platinum, 1 received bleomycin, ifosfamide and cisplatin, 1 received bleomycin, vincristine and cisplatin, 1 received temozolomide and Xeloda, 3 received docetaxel and platinum and 3 received TP and EP successively.

\subsection{Survival outcomes}

The median OS and PFS times were 33.23 months and 22.8 months, respectively, and the 5year OS and PFS rates were $48.5 \%$ and $42.4 \%$, respectively. At the end of follow-up (7th June 2020), 100 patients had experienced cancer recurrence and 92 patients had died. The five-year survival rates of patients with stage I, II, III, and IV disease were $74.8 \%, 56.2 \%, 41.4 \%$ and $0 \%$, respectively. The five-year progression-free survival rates of stage I, II, III, and IV disease were $63.8 \%, 54.5 \%, 24.6 \%$, and $0 \%$, respectively. The survival curves for different FIGO stages are shown in Fig. 3. A comparison of OS and PFS revealed that the prognosis of stage I and stage II tumors classified based on the FIGO 2018 staging system tended to be better than that of stage I and stage II tumors classified based on the FIGO 2008 staging system, and the prognosis of stage III tumors classified with the 2018 staging system was worse than that of the corresponding tumors classified based on the FIGO 2008 staging system. However, these differences were not statistically significant.

Among the entire series $(n=172), 100$ patients experienced cancer recurrence, of which 98 had distant metastasis and 23 had local recurrence. The lung (50/98), liver (42/98), pelvics, and retroperitoneal, mediastinal and supraclavicular lymph nodes (32/98) were the most common sites of metastasis. In addition, 4 patients had brain metastases.

\subsection{Prognostic factors}

As data on the depth of parametrial extension, lymphovascular invasion, depth of stromal invasion, perineural invasion, neoadjuvant therapy and postoperative adjuvant radiotherapy were limited to patients who received surgery, these factors were not included in the univariate analyses of individuals with stages I-IV disease. We performed an additional analysis of the patients who underwent surgery. In the entire series $(n=172)$, multivariate analyses revealed that 
191 advanced FIGO stage ( $p=0.006)$, age ( $\leq 45$ versus $>45$ years: $62.5 \%$ versus $35.0 \% ; p=0.04)$ and 192 tumor size ( $<4 \mathrm{vs} \geq 4 \mathrm{~cm}: 76.0 \%$ versus $39.0 \% ; p=0.013$ ) were significant prognostic factors for 193 OS. In addition, FIGO stage $(p<0.001)$, para-aortic lymph node metastasis (negative vs positive: $19445.9 \%$ vs $6.7 \% ; p=0.014)$ and larger tumor size ( $<4$ vs $\geq 4 \mathrm{~cm}: 64.9 \%$ versus $33.7 \% ; p=0.015)$ 195 were significant prognostic factors for PFS. The 5-year survival rates of patients with small cell 196 carcinoma, large cell carcinoma and typical carcinoid tumors were $49.4 \%, 27.8 \%, 50.0 \%$, and 197 the 5-year disease-free survival rates were $43.2 \%, 33.3 \%$ and $25.0 \%$, respectively. Histological 198 type was not a prognostic factor. Moreover, histological homology, chemotherapy regimen and 199 number of cycles of chemotherapy were not prognostic factors for NETs (Table 2). The survival

200

201

202

203

204 205

206 207 208 209 210 211

212

213

214 215 216 217 218 219 220 curves for patients with different tumor sizes, ages, and para-aortic lymph node statuses are shown in Fig. 4.

For the patients who underwent surgery $(n=108)$, pelvic lymph node metastasis was significantly associated with both OS and PFS in the multivariate analysis (Table 3). Those without pelvic lymph node metastasis had better 5-year OS $(68.0 \%$ versus $41.6 \%, p=0.0028)$ and 5-year PFS (62.6\% versus 29.3\%, $p=0.019)$ rates than those with pelvic lymph node metastasis.

The survival curves for patients with different pelvic lymph node statuses are shown in Fig. 5. In addition, larger tumor size ( $<4 \mathrm{vs} \geq 4 \mathrm{~cm}$ : $80.1 \%$ versus $49.0 \%$; $p=0.02$ ) was a significant prognostic factor for OS. However, histological type, age, histological homology, parametrial involvement, lymphovascular invasion, depth of stromal invasion, perineural invasion, neoadjuvant therapy, adjuvant radiotherapy, chemotherapy regimen and number of cycles of chemotherapy were not prognostic factors (Table 3).

\subsection{Efficacy of treatment}

In our study, patients in the early stage (stages I-IIA2) who received primary surgery tended to have better 5-year OS (67.8\% versus 44.4\%, $p=0.199)$ and 5-year PFS $(62.9 \%$ versus $33.3 \%$, $p=0.113$ ) rates than those who received primary chemoradiation, but the difference was not statistically significant. The 5-year OS and 5-year PFS rates for patients with stage IIB-IIIC2 disease who received primary surgery were $50.6 \%$ and $34.7 \%$, respectively, and the rates for patients who received primary radiotherapy were $42.4 \%$ and $41.3 \%$, respectively. In patients with stage IV disease, primary treatment containing at least six cycles of chemotherapy was associated with significantly better 2 -year OS $(83.3 \%$ versus $9.1 \%, p<0.001)$ and 2 -year PFS 
$221(57.1 \%$ versus $0 \%, p=0.01)$ rates than those primary treatment containing fewer than six cycles 222 of chemotherapy (Table 4).

\subsection{Chemotherapy and toxicity}

224 In our study, the chemotherapy regimen was not a significant prognostic factor for NETs. The 225 5-year OS rates of patients receiving the TP/TC regimen, EP regimen, other regimens and no 226 chemotherapy were $52.1 \%, 49.2 \%, 30.5 \%$ and $40.0 \%$, respectively. And the 5-year DFS rates of 227 patients receiving the TP/TC regimen, EP regimen, other regimens and no chemotherapy were $22841.9 \%, 45.6 \%, 23.8 \%$ and 40.0\%, respectively. The differences were not statistically significant. 229 We further studied the toxicity of the TP/TC regimen and EP regimen. The worst levels of 230 toxicity reached at any time were recorded using the Common Terminology Criteria for Adverse 231 Events (Version 5.0). The incidences of myelosuppression, hepatic dysfunction, and 232 gastrointestinal reactions were 78.5\% (62/79), 27.8\% (22/79) and 13.9\% (11/79), respectively, in 233 patients who received the TP regimen and 75.0\% (57/76), 22.4\% (17/76), and 10.5\%

234 (8/76),respectively, in patients who received the EP regimen. Furthermore, the incidence of grade 235 3-4 toxicity was $45.6 \%$ (36/79) in patients who received the TP regimen and 44.7\% (34/76) in 236 patients who received the EP regimen. There were no significant differences in the above 237 comparisons (Table 5). We believe that the two chemotherapy regimens have similar toxicity.

\section{Discussion}

240 To the best of our knowledge, this is the largest single-center retrospective study of

241 neuroendocrine tumors of the uterine cervix. We studied the prognostic factors and outcomes of 242172 patients with NETs based on the FIGO 2018 staging system. We discussed the appropriate 243 primary treatment for each FIGO stage and evaluated the effect of adjuvant chemotherapy, 244 adjuvant radiotherapy and neoadjuvant chemotherapy. In multivariate analysis, FIGO stage, 245 older age and large tumors were independent prognostic factors for OS. In addition, FIGO stage, 246 para-aortic lymph node metastasis and large tumors were independent prognostic factors for 247 PFS. The TP/TC and EP regimens were the most commonly used regimens and had similar 248 efficacy and toxicity.

\section{$249 \quad 4.1$ FIGO stage and outcomes}

250 At present, the prognostic factors for cervical neuroendocrine carcinoma are controversial; 251 however, the FIGO stage is commonly recognized as an independent prognostic factor. In our 
252 study, the 5-year OS rates of patients with stage I, II, III, and IV disease were $74.8 \%, 56.2 \%$, $25341.4 \%$ and 0\%, respectively, according to the FIGO 2018 staging system, and the 5-year PFS 254 rates were $63.8 \%, 54.5 \%, 30.8 \%$ and $0 \%$, respectively. Wang et al. reported that the 5-year 255 cancer-specific survival rate was $51.5 \%$ in patients with stage I-IIA disease $(\mathrm{n}=123)$ and $24.9 \%$ 256 in patients with stage IIB-IVB disease $(n=56)$ (Wang et al. 2012). Intaraphet et al. reported a rate 257 of $63 \%$ in patients with stage I disease $(n=71), 54 \%$ in patients with stage IIA disease $(n=11)$, $25826 \%$ in patients with stage IIB disease $(n=26)$, and $0 \%$ in patients with stage III and IV disease 259 ( $\mathrm{n}=22)$ (Intaraphet et al. 2014). The 5-year OS and 5-year PFS rates in our study were slightly 260 better than those reported in previous studies. This better survival rate can be explained by our 261 multimodality treatment. Upon the exclusion of 5 patients with stage IVB disease who received 262 palliative chemotherapy, 98.2\% (164/167) received two or more types of treatments. More than $26397.1 \%(165 / 172)$ of patients with stage I to IV disease received chemotherapy, with an average 264 of 4.35 cycles of chemotherapy. We believe that standard and complete multimodality treatment 265 may improve the prognosis of NETs.

\section{4.2 Prognostic factors}

267 Older age was associated with poor survival in several studies(Chen et al. 2008; Intaraphet et 268 al. 2014; Lee et al. 2008; Zhou et al. 2016b). Hoskins found that an age $<50$ years predicted 269 prolonged $\operatorname{OS}(p=0.02)$ (Hoskins et al. 2003). Our study revealed that patients aged 45 years or 270 younger had a better survival rate than those aged older than 45 years $(62.5 \%$ versus $35.0 \%, p$ $=0.005)$. As shown in previous studies, large tumor size was an important prognostic factor for 272 NETs (Atienza-Amores et al. 2014; Gardner et al. 2011; Liao et al. 2012). Liao et al. conducted a 273 large retrospective study of 293 patients and found that tumor size was indicative of a poor 274 prognosis ( $\geq 4 \mathrm{~cm}$ versus $<4 \mathrm{~cm}, \mathrm{HR}=2.37,95 \% \mathrm{CI}=[1.28,4.36], p=0.006$ ) (Liao et al. 2012). 275 Bermúdez et al. reported that patients with tumors $<4 \mathrm{~cm}$ had better 5-year OS rates than those 276 with tumors $>4 \mathrm{~cm}(76 \%$ versus $18 \%, p<0.05)$ (Bermudez et al. 2001). Our study showed that 277 large tumor size was a significant prognostic factor for both OS and PFS $(\mathrm{p}=0.002$ and $p<0.001)$. 278 With more patients and studies, tumor size may ultimately prove to be an important prognostic 279 factor for NETs.

280 NETs have a high incidence of lymph node metastasis, even early stage disease (Atienza281 Amores et al. 2014; Satoh et al. 2014; Tempfer et al. 2018; Zhou et al. 2016a). Radical 282 hysterectomy and pelvic lymph node dissection are commonly recommended in the primary 
283 treatment of patients with early stage NETs. However, few studies have discussed whether para284 aortic lymph node dissection is essential. Boruta recommended radical surgery for all patients 285 with early stage Nets, including para-aortic lymph node dissection (Boruta et al. 2001). In our 286 study, 59 patients had pelvic lymph node involvement, and 15 patients had para-aortic node 287 involvement at the initial diagnosis. After treatment and follow-up, 32 patients had pelvic, 288 retroperitoneal, mediastinal or supraclavicular lymph node metastasis. Furthermore, we found 289 that lymph node metastasis was an independent prognostic factor for both OS and PFS in 290 patients with NETs who received surgery. Therefore, we recommend routine para-aortic lymph 291 node dissection during radical surgery for patients with NETs, although more research is needed.

292

293

294

295

296

297

298

299

300

301

302

303

304

305

306

307

308

309

310

311

312

313

\subsection{Primary treatment}

Because of the rarity of NETs and the lack of multicenter randomized controlled studies, there is no standard treatment for NETs. Both the Gynecologic Cancer InterGroup (GCIG) and the Society of Gynecologic Oncology (SGO) recommend radical surgery as the primary treatment for patients with early stage disease, while chemoradiation is recommended for patients with advanced stage disease (Gardner et al. 2011; Satoh et al. 2014). Most studies report long-term survival outcomes only for patients with early-stage disease who received radical surgical resection and adjuvant chemotherapy (Chen et al. 2008; Cohen et al. 2010; Lee et al. 2015; Zhou et al. 2016b). Cohen et al. showed that the 5-year disease-specific survival rate for patients with stage I-IIA disease $(\mathrm{n}=169)$ who received radical hysterectomy was $38.2 \%$, which was better than that for those who did not $(23.8 \%)(p<0.001)$ (Cohen et al. 2010). Ishikawa et al. found that the hazard ratio (HR) for death after definitive radiotherapy to death after radical surgery was 4.74 (95\% confidence interval [CI], 1.01-15.90) (Ishikawa et al. 2018). However, Chen et al. suggested that the survival outcomes after primary radiotherapy (RT) are superior to those after primary surgery (Chen et al. 2015). The authors attributed this hypothesis to the fact that surgical trauma may increase the number of circulating tumor cells (Khan et al. 2013). In our study, patients in the early stage (stages I-IIA2) who received primary surgery tended to have better 5year OS rates and 5-year PFS rates than those who received primary chemoradiation, but the difference was not statistically significant. Primary surgery with adjuvant chemotherapy or chemoradiotherapy could be the optimal therapy for patients with early stage NETs.

Chemoradiotherapy is commonly recommended in the primary treatment of patients with advanced-stage NETs. Interestingly, we found that the survival outcomes of patients with 
314 advanced-stage disease who received primary radiotherapy were similar to those of patients who 315 received primary surgery. The 5-year OS and 5-year PFS rates for patients with stage IIB-IIIC2 316 disease who received primary radiotherapy were $42.4 \%$ and $41.3 \%$, respectively, and the rates

317 for patients who received primary surgery were $50.6 \%$ and $34.7 \%$, respectively. Surgery may 318 also be an effective treatment for patients with advanced-stage NETs who are not sensitive to 319 radiotherapy.

320 To the best of our knowledge, this is the first study to discuss the association between the 321 number of cycles of chemotherapy and prognosis for patients with stage IV disease. In our study, 322 primary treatment consisting of at least six cycles of chemotherapy was associated with 323 significantly better 2 -year OS $(83.3 \%$ versus $9.1 \%, p<0.001)$ and 2 -year PFS $(57.1 \%$ versus $0 \%$, $324 p=0.01)$ rates than primary treatment consisting of fewer than six cycles of chemotherapy.

325 Similar to small cell lung carcinoma, neuroendocrine cervical carcinoma is sensitive to 326 chemotherapy, and chemotherapy can improve patient prognosis(Atienza-Amores et al. 2014; 327 Gardner et al. 2011; Satoh et al. 2014). Moreover, patients who received more than 6 cycles of 328 chemotherapy in our study had larger tumor sizes $(4.39 \pm 0.6 \mathrm{~cm}$ vs $6.09 \pm 2.2 \mathrm{~cm}, p=0.083)$.

329 Larger tumors are usually associated with greater tumor burden and worse prognosis. In addition, 330 the patients who received more than 6 cycles of chemotherapy were younger $(52 \pm 12.3$ years vs $33147.0 \pm 9.1, p=0.329)$, but the difference was not statistically significant. Cohen found that 332 chemotherapy (as primary treatment or adjuvant or with concurrent radiation) was associated 333 with improved survival in stage IIB-IVA disease compared with treatment without chemotherapy 334 (3-year survival: $17.8 \%$ vs $12.0 \%$; $p=0.043$ ) (Cohen et al. 2010). Similarly, Wang reported that 335 concurrent chemoradiation with EP for at least 5 cycles was associated with even better 5 -year 336 FFS $(62.5 \%$ versus $13.1 \%, p=0.025)$ and CSS $(75.0 \%$ versus $16.9 \%, p=0.016)$ than other 337 treatments (Wang et al. 2012). An adequate number of cycles of chemotherapy seems to be 338 indispensable for patients with stage IV disease. Due to the small number of cases in this study, 339 further studies are needed to prove this finding.

\section{$340 \quad 4.4$ Adjuvant treatment}

341 Given the aggressive behavior of NETs, the use of adjuvant chemotherapy as a part of 342 multimodality treatment has been commonly assessed. The 3-year distant recurrence-free 343 survival rate was $83 \%$ for patients who received chemotherapy and $0 \%$ for patients who did not $344(p=0.025)$ (Zivanovic et al. 2009). Intaraphet et al. reported a 5 -year survival rate of $74.4 \%$ for 
345 patients who received adjuvant chemotherapy, a rate of $55.6 \%$ for those who received surgery 346 alone, a rate of $53.3 \%$ for those who received adjuvant radiotherapy and a rate of $30.1 \%$ for those 347 who received adjuvant chemoradiation ( $p=0.041$ ) (Intaraphet et al. 2014). In our study, adjuvant 348 chemotherapy was used in most patients $(n=105)$ who underwent surgery $(n=108)$ and may be 349 one of the reasons for their good prognosis.

350 In contrast, the benefit of adjuvant radiotherapy for NETs is controversial. Most authors have 351 found that adjuvant radiotherapy does not improve survival. Lee et al. found that patients who 352 received adjuvant radiotherapy had poorer 5-year survival rates than those who did not $(45.5 \%$ 353 vs $52.5 \%, \mathrm{p}=0.37$ ). Zhou et al. reported that the 5-year CSS rates of patients who received radical 354 surgery, radical surgery combined with radiotherapy, and radiotherapy alone were $67.9 \%$, $35549.7 \%$, and $32.6 \%$, respectively $(p<0.001)$ (Zhou et al. 2016b). However, some authors have 356 suggested that adjuvant radiotherapy reduces the local recurrence rate (Chen et al. 2015; 357 Viswanathan et al. 2004). Viswanathan et al. reported that only 2 of 15 patients with NETs who 358 received radiotherapy experienced recurrence in the radiation field, while 2 of 6 patients 359 experienced recurrence after radical surgery without adjuvant radiotherapy (Viswanathan et al. 360 2004). Owing to the aggressive behavior of these tumors, treatment failure usually results in 361 distant metastasis rather than local recurrence (Atienza-Amores et al. 2014; Satoh et al. 2014). In 362 our study, among the entire series ( $n=172), 100$ patients experienced cancer recurrence, of whom 36398 had distant metastasis and 23 had local recurrence. Due to widespread hematogenous 364 365 metastases, recurrence usually occurs outside the radiation field and may explain why adjuvant radiotherapy does not improve the outcomes of patients with NETs.

366 The GCIG and SGO recommend neoadjuvant chemotherapy (NAC) for patients with large 367 tumors $(>4 \mathrm{~cm})$. However, few reports have indicated the benefit of neoadjuvant chemotherapy 368 (Bermudez et al. 2001; Chang et al. 1999). Complete response has been observed in 6/7 patients 369 with NETs after NAC (Chang et al. 1999), but the number of patients is so small that it is 370 difficult to draw a definitive conclusion. Some authors found no improvement in overall survival 371 among patients who received NAC (Dongol et al. 2014; Wang et al. 2012). In our study, 372 neoadjuvant chemotherapy was not a prognostic factor. We found that the 70 patients who 373 received NAC and the other 38 patients who did not receive NAC had similar 5-year OS and 5374 year PFS rates. The 5-year OS rates were $61.1 \%$ and $57.1 \%(p=0.559)$, respectively, and the 5- 
375 year PFS rates were 53.5\% and 51.8\% $(p=0.511)$, respectively. More studies are needed to prove 376 the benefit of NAC.

\section{4.5 Chemotherapy regimen}

378 There is no standard chemotherapy regimen for NETs yet. Etoposide and

379 cisplatin/carboplatin (EP) are the most commonly used regimens in the treatment of NETs

380 (Atienza-Amores et al. 2014; Ishikawa et al. 2019; Lee et al. 2015; Pei et al. 2017; Tempfer et al.

381 2018). However, vincristine, Adriamycin and cyclophosphamide (VAC), irinotecan and platinum

382 (CPT-P), paclitaxel and cisplatin/carboplatin (TC/TP) and various other chemotherapy regimens

383 are also used. Pei et al. found that at least 5 cycles of adjuvant chemotherapy with EP ( $\mathrm{n}=39$ ) was

384 associated with better 5-year recurrence-free survival rates than other treatments $(\mathrm{n}=46)(67.6 \%$

385 vs. 20.9\%, $p<0.001$ ) (Pei et al. 2017). Zivanovic et al. reported that the 3-year recurrence-free

386 survival rate was $83 \%$ for early stage patients who received chemotherapy and $0 \%$ for early stage

387 patients who did not receive chemotherapy as part of their initial treatment $(\mathrm{p}=0.025)$ (Zivanovic

388 et al. 2009). However, Lei et al. reported that patients who received the TC regimen had better 5-

389 year OS and 5-year DFS rates than those who received the non-TC regimen $(p=0.04)$. In our

390 study, the chemotherapy regimen was not a significant prognostic factor for NETs. We found no

391 significant difference in 5-year OS and 5-year PFS between patients receiving the TP/TC

392 regimen and patients receiving the EP regimen or other regimens. More studies are urgently

393 needed to find an appropriate chemotherapy regimen for NETs.

\section{Conclusions}

395 Because of the rarity of NETs, most studies on NETs have been perfomed with small samples 396 or were case reports. To the best of our knowledge, three larger retrospective studies including 397188,193 and 179 patients have been published, exclusive of meta-analyses and studies based on 398 the Surveillance, Epidemiology and End-Results (SEER) program (Cohen et al.2010; Ishikawa et 399 al. 2019; Wang et al. 2012). Our study is the largest single-center study with the fourth largest 400 number of cases. We studied the prognostic factors and outcomes of NETs in 172 patients based 401 on the FIGO 2018 staging system. We discussed the appropriate primary treatment for each 402 FIGO stage and evaluated the efficacy of adjuvant chemotherapy, adjuvant radiotherapy and 403 neoadjuvant chemotherapy. Moreover, we compared the chemotherapy regimens, and further 404 analyzed the toxicities of the two most commonly used regimens. Notably, we found that 405 patients with stage IV disease whose primary treatment included at least six cycles of 
406 chemotherapy had significantly better 2-year OS and 2-year PFS rates than those whose primary 407 treatment included fewer than six cycles of chemotherapy. However, there are some limitations 408 in this study. First, this study was retrospective; therefore, selection bias is unavoidable. Second, 409 para-aortic LN metastasis was an important prognostic factor in our study, but only 12 of 108 410 patients underwent para-aortic lymph node dissection. Finally, this was a single-centre study, and 411 the sample size was relatively small, especially regarding the number of LCNECs and carcinoids. 412 More studies with larger cohorts are needed.

413 In conclusion, advanced FIGO stage, large tumor, older age and lymph node metastasis are 414 independent prognostic factors for NETs of the cervix. Adjuvant chemotherapy, adjuvant 415 radiotherapy and neoadjuvant chemotherapy did not improve the outcomes of patients with 416 NETs. The TP/TC and EP regimens were the most commonly used regimens, with similar 417 efficacies and toxicities. Standardized and complete multimodality treatment may improve the 418 survival of patients with NETs.

419

\section{Acknowledgements}

We thank Xiaojie Wang for helpful suggestions and comments on the manuscipt.

422

\section{References}

Albores-Saavedra J, Gersell D, Gilks CB, Henson DE, Lindberg G, Santiago H, Scully RE, Silva E, Sobin LH, Tavassoli FJ, Travis WD, and Woodruff JM. 1997. Terminology of endocrine tumors of the uterine cervix: results of a workshop sponsored by the College of American Pathologists and the National Cancer Institute. Arch Pathol Lab Med 121:34-39.

Atienza-Amores M, Guerini-Rocco E, Soslow RA, Park KJ, and Weigelt B. 2014. Small cell carcinoma of the gynecologic tract: a multifaceted spectrum of lesions. Gynecol Oncol 134:410-418. 10.1016/j.ygyno.2014.05.017

Bermudez A, Vighi S, Garcia A, and Sardi J. 2001. Neuroendocrine cervical carcinoma: a diagnostic and therapeutic challenge. Gynecol Oncol 82:32-39. 10.1006/gyno.2001.6201

Boruta DM, 2nd, Schorge JO, Duska LA, Crum CP, Castrillon DH, and Sheets EE. 2001. Multimodality therapy in early-stage neuroendocrine carcinoma of the uterine cervix. Gynecol Oncol 81:82-87. 10.1006/gyno.2000.6118

Chang TC, Hsueh S, Lai CH, Tseng CJ, Lee KF, Huang KG, Chou HH, and Soong YK. 1999. Phase II trial of neoadjuvant chemotherapy in early-stage small cell cervical cancer. Anticancer Drugs 10:641-646. 10.1097/00001813-199908000-00003

Chen J, Macdonald OK, and Gaffney DK. 2008. Incidence, mortality, and prognostic factors of small cell carcinoma of the cervix. Obstet Gynecol 111:1394-1402. 10.1097/AOG.0b013e318173570b

Chen TC, Huang HJ, Wang TY, Yang LY, Chen CH, Cheng YM, Liou WH, Hsu ST, Wen KC, Ou YC, Hung YC, Lai HC, Ho CM, and Chang TC. 2015. Primary surgery versus primary radiation therapy for FIGO stages III small cell carcinoma of the uterine cervix: A retrospective Taiwanese Gynecologic Oncology Group study. Gynecol Oncol 137:468-473. 10.1016/j.ygyno.2015.03.015

Cohen JG, Kapp DS, Shin JY, Urban R, Sherman AE, Chen LM, Osann K, and Chan JK. 2010. Small cell carcinoma of the cervix: treatment and survival outcomes of 188 patients. Am J Obstet Gynecol 203:347 e341-346. 10.1016/j.ajog.2010.04.019 
448

449

450

451

452

453

454

455

456

457

458

459

460

461

462

463

464

465

466

467

468

469

470

471

472

473

474

475

476

477

478

479

480

481

482

483

484

485

486

487

488

489

490

491

492

493

494

495

496

497

498

499

500

501

502

503

Conner MG, Richter H, Moran CA, Hameed A, and Albores-Saavedra J. 2002. Small cell carcinoma of the cervix: a clinicopathologic and immunohistochemical study of 23 cases. Ann Diagn Pathol 6:345-348. 10.1053/adpa.2002.36661

Dongol S, Tai Y, Shao Y, Jiang J, and Kong B. 2014. A retrospective clinicopathological analysis of small-cell carcinoma of the uterine cervix. Mol Clin Oncol 2:71-75. 10.3892/mco.2013.193

Gardner GJ, Reidy-Lagunes D, and Gehrig PA. 2011. Neuroendocrine tumors of the gynecologic tract: A Society of Gynecologic Oncology (SGO) clinical document. Gynecol Oncol 122:190-198. 10.1016/j.ygyno.2011.04.011

Hoskins PJ, Swenerton KD, Pike JA, Lim P, Aquino-Parsons C, Wong F, and Lee N. 2003. Small-cell carcinoma of the cervix: fourteen years of experience at a single institution using a combined-modality regimen of involved-field irradiation and platinum-based combination chemotherapy. $J$ Clin Oncol 21:3495-3501. 10.1200/JCO.2003.01.501

Intaraphet S, Kasatpibal N, Siriaunkgul S, Chandacham A, Sukpan K, and Patumanond J. 2014. Prognostic factors for small cell neuroendocrine carcinoma of the uterine cervix: an institutional experience. Int J Gynecol Cancer 24:272-279. 10.1097/IGC.0000000000000059

Ishikawa M, Kasamatsu T, Tsuda H, Fukunaga M, Sakamoto A, Kaku T, Kato T, Takahashi K, Ariyoshi K, Suzuki $\mathrm{K}$, Arimoto T, Matsumoto Y, Nakai H, Inoue T, Yokoyama M, Kawabata T, Kodama S, Miyamoto T, Takano M, and Yaegashi N. 2019. A multi-center retrospective study of neuroendocrine tumors of the uterine cervix: Prognosis according to the new 2018 staging system, comparing outcomes for different chemotherapeutic regimens and histopathological subtypes. Gynecol Oncol 155:444-451. 10.1016/j.ygyno.2019.09.018

Ishikawa M, Kasamatsu T, Tsuda H, Fukunaga M, Sakamoto A, Kaku T, Nakanishi T, Hasumi Y, Iwata T, Baba T, Nogawa T, Kudaka W, Kaneda H, Ono S, Saito F, Taniguchi Y, Okada S, Mizuno M, Onda T, and Yaegashi N. 2018. Prognostic factors and optimal therapy for stages I-II neuroendocrine carcinomas of the uterine cervix: A multi-center retrospective study. Gynecol Oncol 148:139-146. 10.1016/j.ygyno.2017.10.027

Khan MS, Kirkwood A, Tsigani T, Garcia-Hernandez J, Hartley JA, Caplin ME, and Meyer T. 2013. Circulating tumor cells as prognostic markers in neuroendocrine tumors. $J$ Clin Oncol 31:365-372. 10.1200/JCO.2012.44.2905

Lee JM, Lee KB, Nam JH, Ryu SY, Bae DS, Park JT, Kim SC, Cha SD, Kim KR, Song SY, and Kang SB. 2008. Prognostic factors in FIGO stage IB-IIA small cell neuroendocrine carcinoma of the uterine cervix treated surgically: results of a multi-center retrospective Korean study. Ann Oncol 19:321-326. 10.1093/annonc/mdm465

Lee SW, Lim KT, Bae DS, Park SY, Kim YT, Kim KR, and Nam JH. 2015. A multicenter study of the importance of systemic chemotherapy for patients with small-cell neuroendocrine carcinoma of the uterine cervix. Gynecol Obstet Invest 79:172-178. 10.1159/000367920

Liao LM, Zhang X, Ren YF, Sun XY, Di N, Zhou N, Pan RK, Ma SH, and Zhou LX. 2012. Chromogranin A (CgA) as poor prognostic factor in patients with small cell carcinoma of the cervix: results of a retrospective study of 293 patients. PLoS One 7:e33674. 10.1371/journal.pone.0033674

Pei X, Xiang L, Ye S, He T, Cheng Y, Yang W, Wu X, and Yang H. 2017. Cycles of cisplatin and etoposide affect treatment outcomes in patients with FIGO stage I-II small cell neuroendocrine carcinoma of the cervix. Gynecol Oncol 147:589-596. 10.1016/j.ygyno.2017.09.022

Satoh T, Takei Y, Treilleux I, Devouassoux-Shisheboran M, Ledermann J, Viswanathan AN, Mahner S, Provencher DM, Mileshkin L, Avall-Lundqvist E, Pautier P, Reed NS, and Fujiwara K. 2014. Gynecologic Cancer InterGroup (GCIG) consensus review for small cell carcinoma of the cervix. Int J Gynecol Cancer 24:S102-108. 10.1097/IGC.0000000000000262

Tempfer CB, Tischoff I, Dogan A, Hilal Z, Schultheis B, Kern P, and Rezniczek GA. 2018. Neuroendocrine carcinoma of the cervix: a systematic review of the literature. BMC Cancer 18:530. 10.1186/s12885-0184447-x

Viswanathan AN, Deavers MT, Jhingran A, Ramirez PT, Levenback C, and Eifel PJ. 2004. Small cell neuroendocrine carcinoma of the cervix: outcome and patterns of recurrence. Gynecol Oncol 93:27-33. 10.1016/j.ygyno.2003.12.027

Wang KL, Chang TC, Jung SM, Chen CH, Cheng YM, Wu HH, Liou WS, Hsu ST, Ou YC, Yeh LS, Lai HC, Huang CY, Chen TC, Chang CJ, and Lai CH. 2012. Primary treatment and prognostic factors of small cell neuroendocrine carcinoma of the uterine cervix: a Taiwanese Gynecologic Oncology Group study. Eur J Cancer 48:1484-1494. 10.1016/j.ejca.2011.12.014

Peer) reviewing PDF | (2020:10:53954:3:0:NEW 8 May 2021) 
504

505

506

507

508

509

510

511

512

513

514

515

516

517
Zhou J, Sun JY, Chen SY, Li FY, Lin HX, Wu SG, and He ZY. 2016a. Prognostic value of lymph node ratio in patients with small-cell carcinoma of the cervix based on data from a large national registry. Onco Targets Ther 9:67-73. 10.2147/OTT.S96206

Zhou J, Yang HY, Wu SG, He ZY, Lin HX, Sun JY, Li Q, and Guo ZW. 2016b. The local treatment modalities in FIGO stage I-II small-cell carcinoma of the cervix are determined by disease stage and lymph node status. Cancer Med 5:1108-1115. 10.1002/cam4.687

Zivanovic O, Leitao MM, Jr., Park KJ, Zhao H, Diaz JP, Konner J, Alektiar K, Chi DS, Abu-Rustum NR, and Aghajanian C. 2009. Small cell neuroendocrine carcinoma of the cervix: Analysis of outcome, recurrence pattern and the impact of platinum-based combination chemotherapy. Gynecol Oncol 112:590-593. 10.1016/j.ygyno.2008.11.010 


\section{Figure 1}

HE staining and immunohistochemistry of neuroendocrine tumors of the uterine cervix .

Hematoxylin and eosin staining showed the obvious morphological characteristics of small cell carcinoma and large cell carcinoma of the cervix.Immunohistochemistry showed that CD56, chromogranin $\mathrm{A}(\mathrm{CgA})$ and synaptophysin (Syn) were positive. 


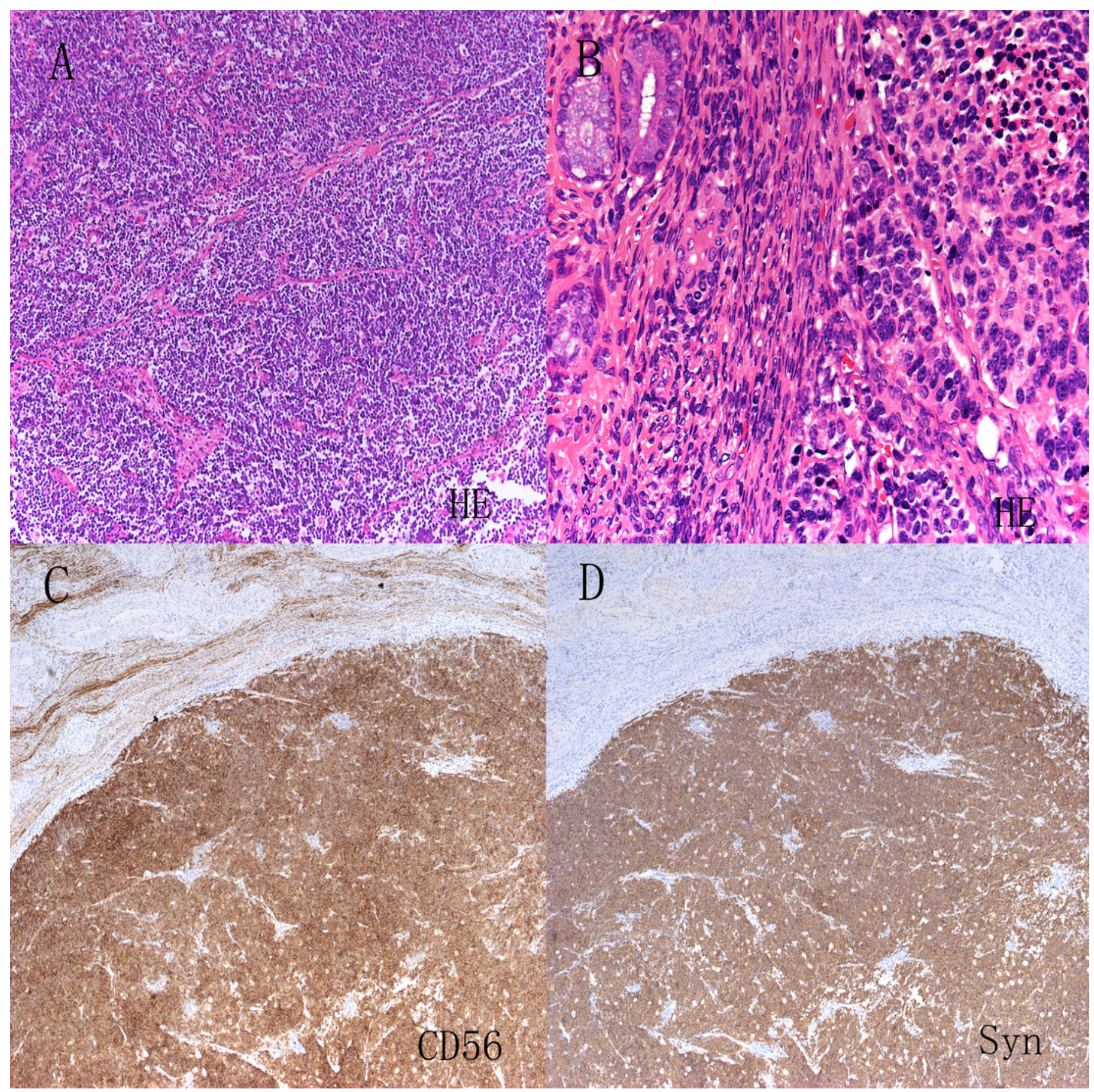




\section{Figure 2}

Patients inclusion/exclusion process and treatment algorithm.

Notes: NACT: neoadjuvant chemotherapy; CCRT: concurrent chemoradiation;

RT:radiotherapy; CT: chemotherapy; EP: etoposide and cisplatin/carboplatin; TP: paclitaxel and cisplatin/carboplatin; Other regimens: 1 patient received paclitaxel liposome, 2 received gemcitabine + platinum, 1 received bleomycin + ifosfamide + cisplatin, 1 received bleomycin + vincristine + cisplatin, 1 received temozolomide + xeloda, 3 received docetaxel + platinum, 3 received TP and EP successively.

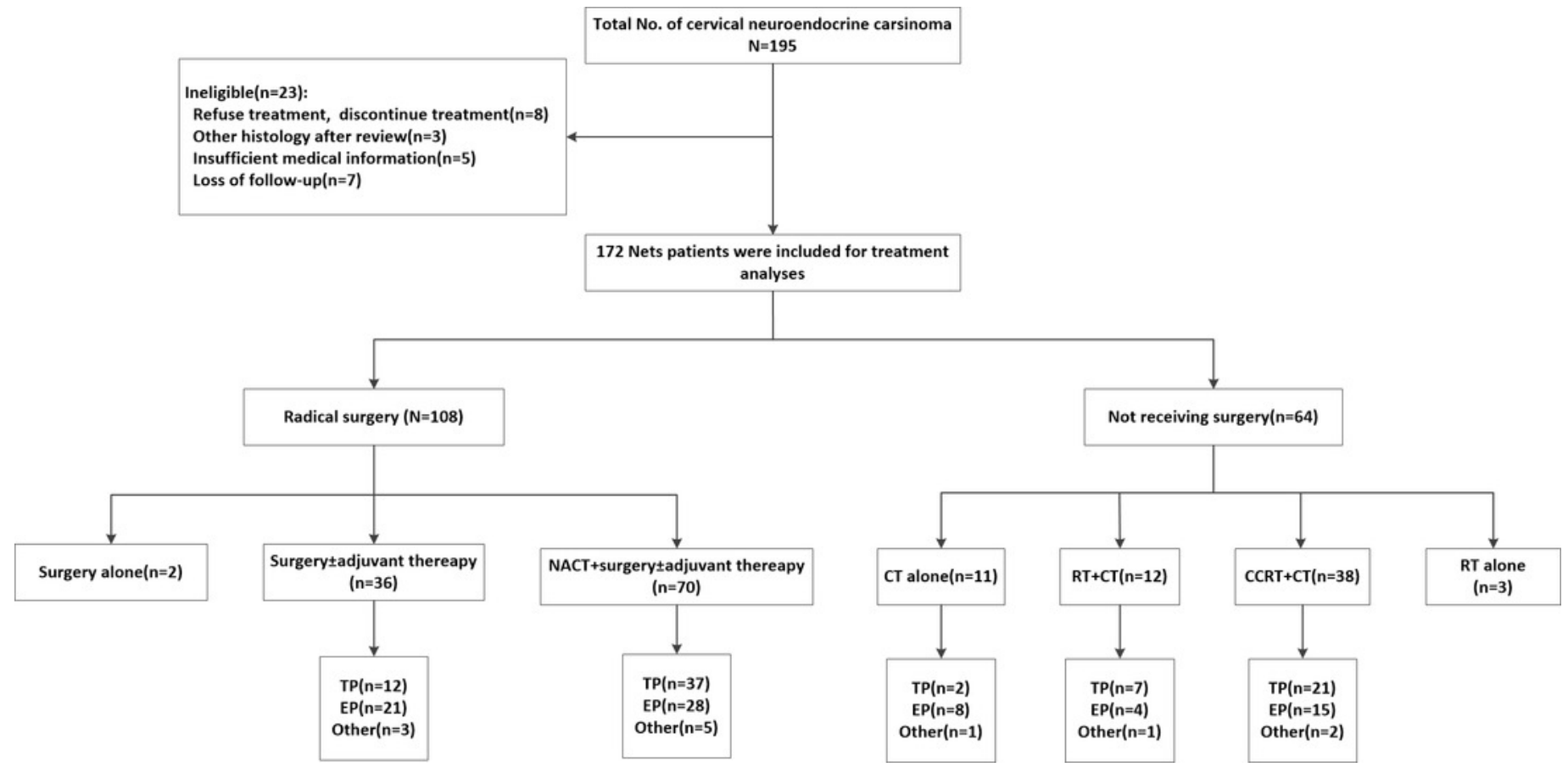


Figure 3

Survival curves of all patients at each stage.(A) OS and (B) PFS.
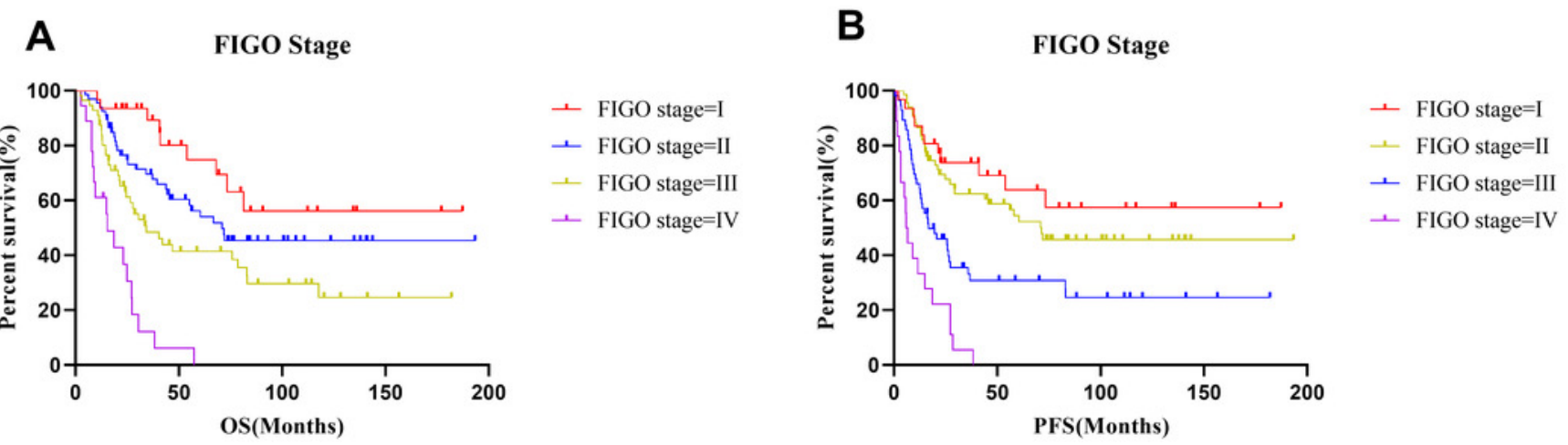
Figure 4

Comparison of survival curves in 172 patients with different tumor size, age and paraaortic lymph node status.(A),(C) and (E) for OS, (B),(D) and (F) for PFS.
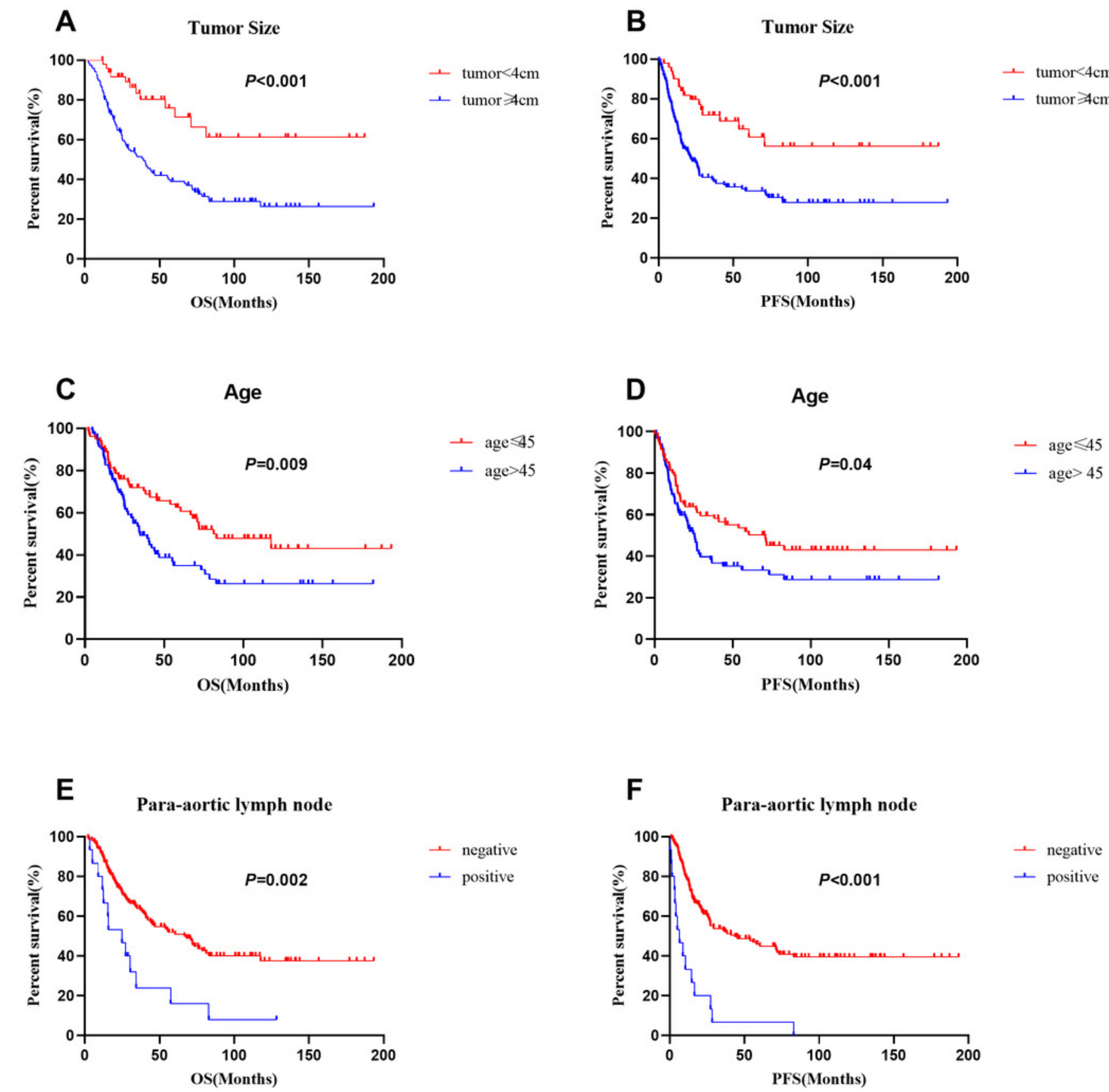

+ tumor $<4 \mathrm{~cm}$
+ tumor $\geqslant 4 \mathrm{~cm}$
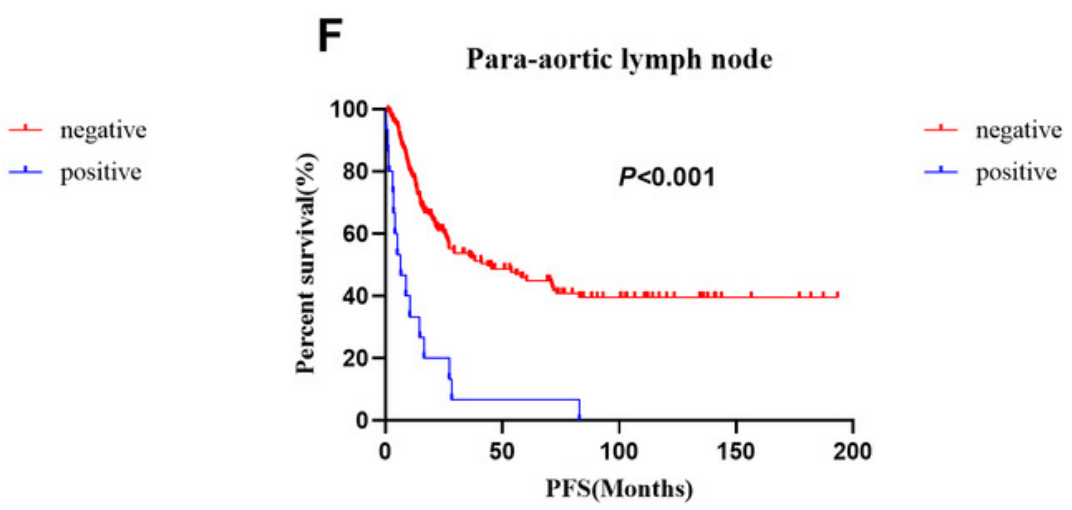
Figure 5

Comparison of survival curves in 108 patients who receiving surgery with dirrerent pelvic lymph node status.(A) OS and (B) PFS.
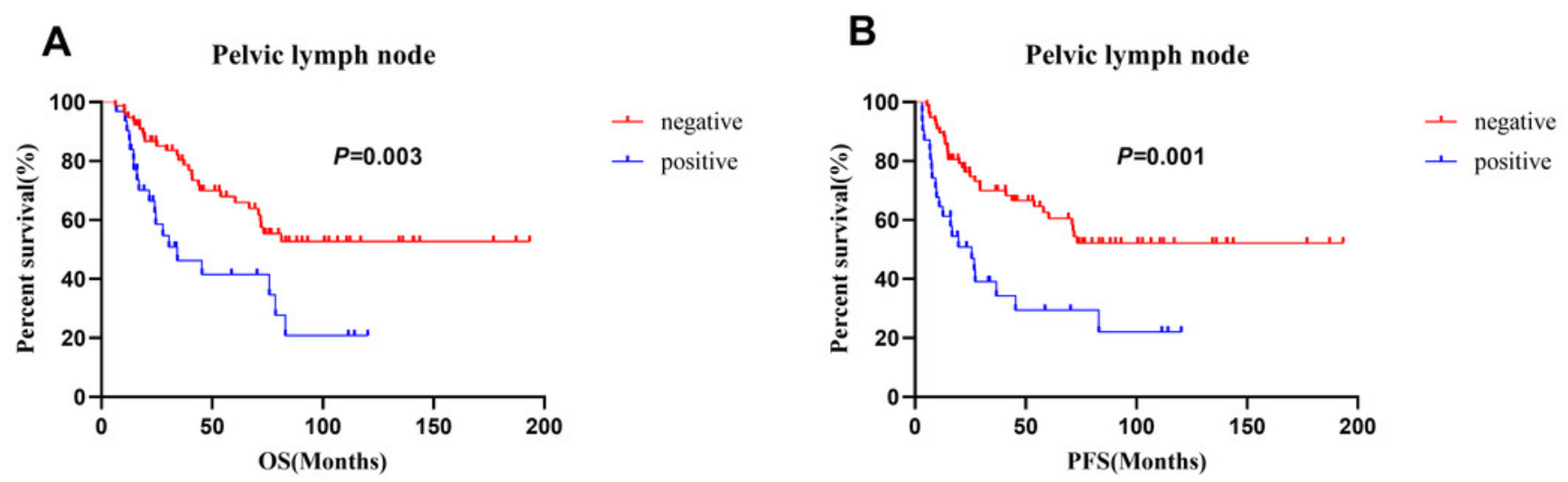


\section{Table $\mathbf{1}$ (on next page)}

Patient, tumour, and treatment characteristics $(\mathrm{N}=172)$.

Notes: LGNET: low grade neuroendocrine tumor; HGNEC: high grade neuroendocrine carcinoma; SCNEC: small cell neuroendocrine carcinoma; LCNEC: large cell neuroendocrine carcinoma; NOT classified:one case included atypucal carcinoid tumor and SCENC at the same time; Adjuvant therapy includes chemotherapy or radiotherapy; FIGO: International Federation of Gynecology and Obstetrics; NACT:neoadjuvant chemotherapy; CCRT: concurrent chemoradiation; CT:chemotherapy; RT:radiotherapy; Other regimens: 1 patient received paclitaxel liposome, 2 received gemcitabine + platinum, 1 received bleomycin + ifosfamide+ cisplatin, 1 received bleomycin + vincristine + cisplatin, 1 received temozolomide + xeloda, 3 received docetaxel + platinum, 3 received TP and EP successively. 


\begin{tabular}{lll}
\hline Variables & No.of & $\%$ \\
patients &
\end{tabular}

Hystological type

Typical carcinoid tumor

SCNEC

LCNEC

Not classified

Histological homology

Pure

Mixed

FIGO stage(2018)

I

IA

IB1

IB2

IB3

II

IIA1

IIA2

IIB

III
4

161

6

1

0.6

126

73.3

46

26.7

2

1.2

2.3

19

11.0

6

3.5

17

9.8

22

12.8

27

15.7 
IIIA

IIIB

IIIC1

IIIC2

IV

IVA

IVB

FIGO stage(2018)

IA-IIA2

IIB-IVB

Age(years)

$\leq 45$

$>45$

Tumor size $(\mathrm{cm})$

$<2$

$2-4$

$4 \leq$

Lymph node metastasis

Pelvic only

Pelvic and para-aortic

Negative

Primary treatment
3

8

38

8

0

18

0.0

10.5

71

41.3

101

58.7

80

46.5

92

53.5

9

5.2

41

23.8

122

70.9

44

25.6

15

8.7

113

65.7 
Surgery+adjuvant therapy

$\mathrm{NACT}+$ surgery \pm adjuvant therapy

Surgery alone

$\mathrm{CCRT}+\mathrm{CT}$

$\mathrm{RT}+\mathrm{CT}$

CT alone

RT alone

Chemotherapy regimen

EP

$\mathrm{TP}$

Other regimens

Without chemotherapy
36

70

2

38

12

11

3

76

79

12

5
20.9

40.7

1.2

22.1

7.0

6.4

1.7

45.9

44.2

7.0

2.9

1 


\section{Table 2 (on next page)}

Prognostic factors for NETs of the uterine cervix $(n=172)$.

Notes: a: one contains both SCNEC and ACT components; LCNEC: large cell neuroendocrine carcinomas; SCNEC: Small cell neuroendocrine carcinoma; FIGO: International; Federation of Gynecology and Obstetrics; LN:lymphnode; EP: etoposide and cisplatin/carboplatin; TP:

paclitaxel and cisplatin/carboplatin; Other regimens: 1 patient received paclitaxel liposome, 2 received gemcitabine + platinum, 1 received bleomycin + ifosfamide+ cisplatin, 1 received bleomycin + vincristine + cisplatin, 1 received temozolomide + xeloda, 3 received docetaxel + platinum, 3 received TP and EP successively. 


\begin{tabular}{|c|c|c|c|c|c|c|c|c|c|c|c|c|c|c|c|}
\hline \multirow{3}{*}{ Variable } & \multirow{3}{*}{$\mathrm{n}$} & \multicolumn{7}{|c|}{ Overall survival } & \multicolumn{7}{|c|}{ Progression free survival } \\
\hline & & \multicolumn{4}{|c|}{ Univariate } & \multicolumn{3}{|c|}{ Multivariate } & \multicolumn{4}{|c|}{ Univariate } & \multicolumn{3}{|c|}{ Multivariate } \\
\hline & & 5 -year OS(\%) & $H R$ & $95 \% \mathrm{Cl}$ & $\mathrm{P}$ & HR & $95 \% \mathrm{Cl}$ & $\mathrm{P}$ & 5-year PFS(\%) & $\mathrm{HR}$ & $95 \% \mathrm{Cl}$ & $\mathrm{P}$ & $\mathrm{HR}$ & $95 \% \mathrm{Cl}$ & $\mathrm{P}$ \\
\hline Hystological type ${ }^{a}$ & & & 1.180 & $0.708-1.967$ & 0.525 & & & & & 1.462 & $0.864-2.473$ & 0.157 & & & \\
\hline Typical carcinoid tumor & 4 & 50.0 & & & & & & & 25.0 & & & & & & \\
\hline LCNEC & 6 & 27.8 & & & & & & & 33.3 & & & & & & \\
\hline SCNEC & 161 & 49.4 & & & & & & & 43.2 & & & & & & \\
\hline Age, years & & & 1.754 & $1.152-2.672$ & 0.009 & 1.569 & $1.021-2.495$ & 0.040 & & 1.521 & $1.019-2.269$ & 0.04 & 1.441 & $0.940-2.210$ & 0.094 \\
\hline$\leq 45$ & 80 & 62.5 & & & & & & & 51.9 & & & & & & \\
\hline$>45$ & 92 & 35.0 & & & & & & & 33.3 & & & & & & \\
\hline Tumor size $(\mathrm{cm})$ & & & 3.198 & $1.743-5.868$ & $<0.001$ & 2.194 & $1.179-4.082$ & 0.013 & & 2.585 & $1.533-4.360$ & $<0.001$ & 1.929 & $1.133-3.282$ & 0.015 \\
\hline$<4$ & 50 & 76.0 & & & & & & & 64.9 & & & & & & \\
\hline$\geq 4$ & 122 & 39.0 & & & & & & & 33.7 & & & & & & \\
\hline FIGO stage(2018) & & & 2.053 & $1.591-2.650$ & $<0.001$ & 1.726 & $1.163-2.404$ & 0.006 & & 2.067 & $1.622-2.633$ & $<0.001$ & 1.731 & $1.316-2.279$ & $<0.001$ \\
\hline 1 & 31 & 74.8 & & & & & & & 63.8 & & & & & & \\
\hline ॥ & 67 & 56.2 & & & & & & & 54.5 & & & & & & \\
\hline III & 56 & 41.4 & & & & & & & 30.8 & & & & & & \\
\hline IV & 18 & 0 & & & & & & & 0 & & & & & & \\
\hline Histological homology & & & 0.927 & $0.569-1.509$ & 0.76 & & & & & 0.860 & $0.543-1.361$ & 0.518 & & & \\
\hline Pure & 126 & 47.4 & & & & & & & 40.2 & & & & & & \\
\hline Mixed & 46 & 52.5 & & & & & & & 49.1 & & & & & & \\
\hline Pelvic LN metastasis & & & 2.544 & $1.681-3.85$ & $<0.001$ & 1.381 & $0.776-2.459$ & 0.273 & & 2.799 & $1.882-4.163$ & $<0.001$ & 1.377 & $0.772-2.456$ & 0.279 \\
\hline
\end{tabular}




\begin{tabular}{|c|c|c|c|c|c|c|c|c|c|c|c|c|c|c|c|}
\hline No & 113 & 59.9 & & & & & & & 55.2 & & & & & & \\
\hline Yes & 59 & 26.8 & & & & & & & 18.1 & & & & & & \\
\hline Para-aortic LN metastasis & & & 2.534 & $1.406-4.567$ & 0.002 & 1.081 & $0.549-2.127$ & 0.822 & & 4.200 & $2.411-7.317$ & $<0.001$ & 2.185 & 1.171-3.977 & 0.014 \\
\hline No & 157 & 51.9 & & & & & & & 45.9 & & & & & & \\
\hline Yes & 15 & 16.0 & & & & & & & 6.7 & & & & & & \\
\hline Chemotherapy regimen & & & 1.221 & $0.943-1.582$ & 0.129 & & & & & 1.145 & $0.883-1.484$ & 0.307 & & & \\
\hline TP & 79 & 52.1 & & & & & & & 41.9 & & & & & & \\
\hline EP & 76 & 49.2 & & & & & & & 45.6 & & & & & & \\
\hline Other & 12 & 30.5 & & & & & & & 23.8 & & & & & & \\
\hline Without chemotheapy & 5 & 40.0 & & & & & & & 40.0 & & & & & & \\
\hline Cycle of chemotherapy & & & 0.679 & $0.439-1.051$ & 0.082 & 0.666 & $0.423-1.049$ & 0.080 & & 0.785 & $0.521-1.181$ & 0.245 & & & \\
\hline $0-5$ & 107 & 45.2 & & & & & & & 40.9 & & & & & & \\
\hline$\geq 6$ & 65 & 54.0 & & & & & & & 45.3 & & & & & & \\
\hline
\end{tabular}




\section{Table 3 (on next page)}

Prognostic factors for patients who underwent surgical treatment $(n=108)$.

Notes: a: one contains both small cell carcinoma and atypical carcinoid tumor components;LCNEC: large cell neuroendocrine carcinomas; SCNEC: Small cell neuroendocrine carcinoma; FIGO: International; Federation of Gynecology and Obstetrics; LN:lymphnode; EP: etoposide and cisplatin/carboplatin; TP: paclitaxel and cisplatin/carboplatin; Other regimens: 1 received gemcitabine + platinum, 1 received bleomycin + ifosfamide+ cisplatin, 1 received bleomycin + vincristine + cisplatin, 3 received docetaxel + platinum, 1 received TP and EP successively. 


\begin{tabular}{|c|c|c|c|c|c|c|c|c|c|c|c|c|c|c|c|}
\hline \multirow{3}{*}{ Variable } & \multicolumn{8}{|c|}{ Overall survival } & \multicolumn{7}{|c|}{ Progression free survival } \\
\hline & \multirow[t]{2}{*}{$n$} & \multicolumn{4}{|c|}{ Univariate } & \multicolumn{3}{|c|}{ Multivariate } & \multicolumn{4}{|c|}{ Univariate } & \multicolumn{3}{|c|}{ Multivariate } \\
\hline & & 5 -year OS(\%) & $H R$ & $95 \% \mathrm{Cl}$ & $P$ & $\mathrm{HR}$ & $95 \% \mathrm{Cl}$ & $P$ & 5-year PFS(\%) & HR & $95 \% \mathrm{Cl}$ & $P$ & $\mathrm{HR}$ & $95 \% \mathrm{Cl}$ & $P$ \\
\hline Typical carcinoid tumor & 2 & 50.0 & & & & & & & 50.0 & & & & & & \\
\hline LCNEC & 5 & 62.4 & & & & & & & 54.2 & & & & & & \\
\hline Age,years & & & 1.612 & $0.903-2.878$ & 0.106 & & & & & 1.564 & $0.901-2.713$ & 0.112 & & & \\
\hline$\leq 45$ & 60 & 69.3 & & & & & & & 61.2 & & & & & & \\
\hline$>45$ & 48 & 47.8 & & & & & & & 43.0 & & & & & & \\
\hline Tumor size $(\mathrm{cm})$ & & & 2.901 & $1.402-6.006$ & 0.004 & 2.440 & $1.154-5.163$ & 0.020 & & 2.176 & $1.160-4.081$ & 0.015 & 1.938 & $0.986-3.809$ & 0.055 \\
\hline$<4$ & 43 & 80.1 & & & & & & & 68.9 & & & & & & \\
\hline FIGO stage(2018) & & & 1.836 & $1.222-2.759$ & 0.003 & 1.275 & $0.587-2.770$ & 0.539 & & 1.895 & $1.271-2.825$ & 0.002 & 1.203 & $0.576-2.512$ & 0.623 \\
\hline 1 & 29 & 72.3 & & & & & & & 64.5 & & & & & & \\
\hline " & 49 & 63.0 & & & & & & & 59.8 & & & & & & \\
\hline III & 29 & 43.8 & & & & & & & 32.7 & & & & & & \\
\hline IV & 1 & 0.0 & & & & & & & 0.0 & & & & & & \\
\hline Histological homology & & & 0.948 & $0.513-1.753$ & 0.866 & & & & & 0.972 & $0.549-1.722$ & 0.924 & & & \\
\hline Pure & 68 & 59.9 & & & & & & & 51.3 & & & & & & \\
\hline Mixed & 48 & 61.8 & & & & & & & 57.4 & & & & & & \\
\hline Pelvic LN metastasis & & & 2.459 & $1.368-4.420$ & 0.003 & 1.969 & $1.076-3.603$ & 0.028 & & 2.572 & $1.471-4.499$ & 0.001 & 2.035 & $1.126-3.676$ & 0.019 \\
\hline
\end{tabular}




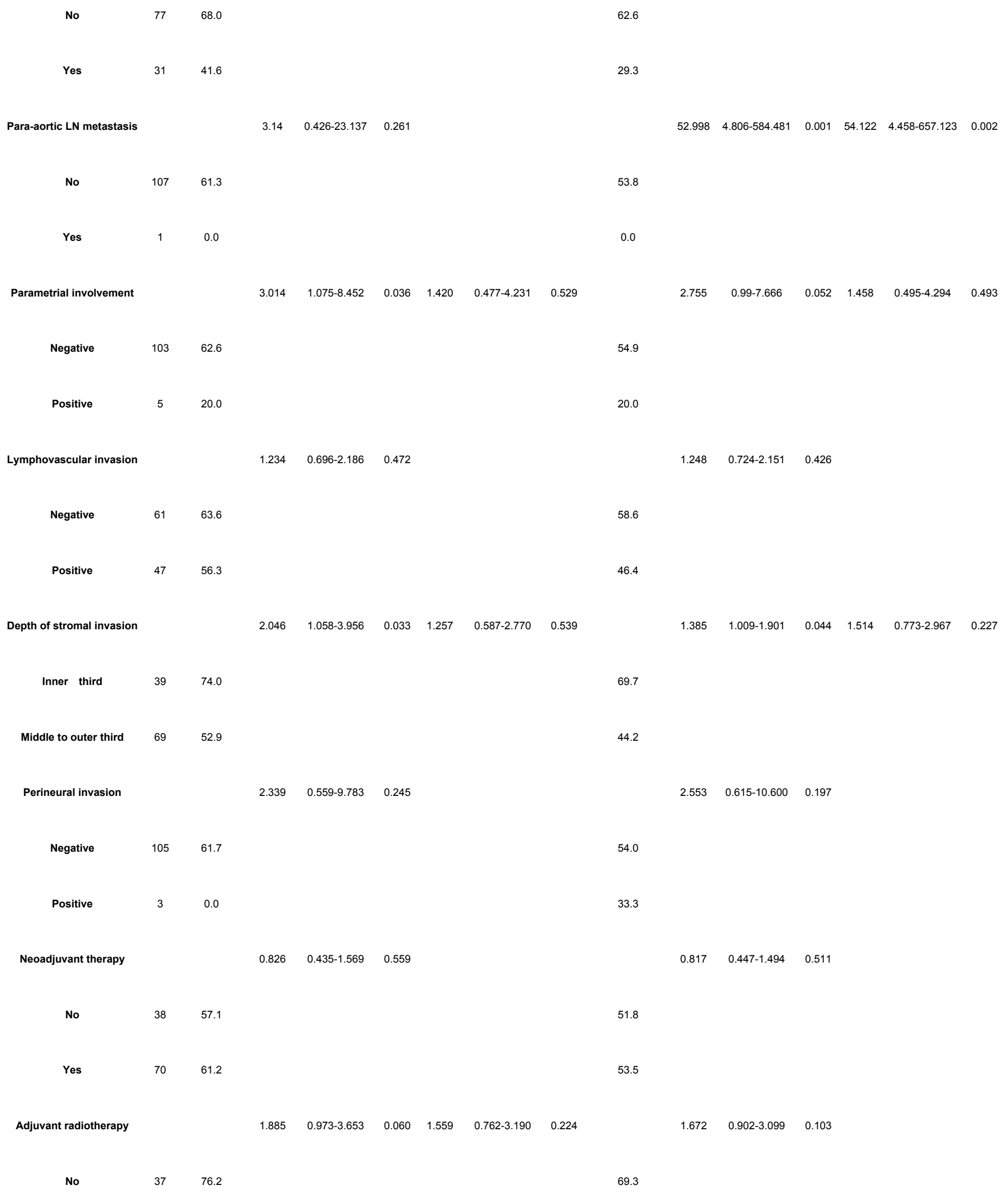


Yes

71

52.4

Adjuvant chemotherapy

No

Yes

105

60.4

Chemotherapy regimen

TP

49

58.0

EP

$49 \quad 66.1$

Other regimens

8

46.7

Without chemotheapy

50.0

Cycle of chemotherapy

0-5

$\geq 6$
$1.184 \quad 0.163-8.595 \quad 0.867$

66.7

$1.009 \quad 0.664-1.535 \quad 0.965$

56.0

50.0

$\begin{array}{lll}0.764 & 0.428-1.363 & 0.362\end{array}$
53.3

36.5

44.3

$\begin{array}{lll}1.471 & 0.203-10.649 & 0.702\end{array}$

66.7

53.0

$1.037 \quad 0.701-1.532 \quad 0.857$

$\begin{array}{lll}0.823 & 0.476-1.423 & 0.485\end{array}$

1 
Table 4 (on next page)

Treatment and outcomes of the patients of NETs.

Notes: RT:radiotherapy; 


\begin{tabular}{|c|c|c|c|c|c|}
\hline Treatment & $\mathrm{N}$ & 5-year OS & $P$ & 5-year PFS & $P$ \\
\hline \multicolumn{6}{|l|}{ Stage I-IIA2(FIGO 2018) } \\
\hline Primary surgery & 62 & 67.8 & 0.199 & 62.9 & 0.113 \\
\hline Primary RT & 9 & 44.4 & & 33.3 & \\
\hline Stage IIB-III(FIGO 2018) & & & 0.673 & & 0.285 \\
\hline Primary surgery & 45 & 50.6 & & 34.7 & \\
\hline Primary RT & 38 & 42.4 & & 41.3 & \\
\hline Stages IVB(FIGO 2018) & $\mathrm{N}$ & 2-year OS & $\mathrm{P}$ & 2-year PFS & $\mathrm{P}$ \\
\hline Cycle of chemotherapy (0-5) & 11 & 9.1 & $<0.001$ & 0.0 & 0.01 \\
\hline Cycle of chemotherapy $>5$ & 7 & 83.3 & & 57.1 & \\
\hline
\end{tabular}

1 


\section{Table 5 (on next page)}

The toxicities of TP and EP regimen. 


\begin{tabular}{|c|c|c|c|c|}
\hline Toxicity & TP & EP & $\chi^{2}$ & $P$ \\
\hline Myelosuppression & & & 0.263 & 0.608 \\
\hline No & 17 & 19 & & \\
\hline Yes & 62 & 57 & & \\
\hline Hepatic dysfunction & & & 0.618 & 0.432 \\
\hline No & 57 & 59 & & \\
\hline Yes & 22 & 17 & & \\
\hline Nausea/vomiting & & & 0.416 & 0.519 \\
\hline No & 68 & 68 & & \\
\hline Yes & 11 & 8 & & \\
\hline Grade 3-4 toxicities & & & 0.011 & 0.917 \\
\hline No & 43 & 42 & & \\
\hline Yes & 36 & 34 & & \\
\hline
\end{tabular}

